\title{
Diabetes and Comorbidities Risk Assessment in Hospitalization and Fatalities from the Mexican COVID-19 Surveillance System
}

Héctor Gallardo-Rincóna , Alejandra Montoyaa , Luis Alberto Martínez-Juárez a,b, Julieta Lomelín-Gascóna, Rodrigo Saucedo-Martíneza, Ricardo Mújica-Rosalesa, Roberto Tapia-Conyera,c

Affiliations:

aCarlos Slim Foundation

bLondon School of Hygiene and Tropical Medicine

cNational Autonomous University of Mexico

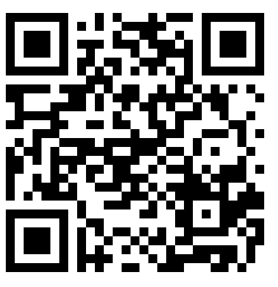

Abstract: 1068-P

American

Diabetes

Association

81 ST SCIENTIFIC

VIRTUAL | JUNE 25-29, 2021

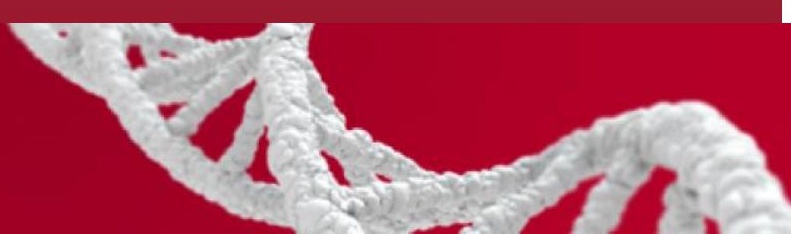




\section{FINANCIAL DISCLOSURE}

\section{Presenter: Héctor Gallardo-Rincón ${ }^{a}$}

\section{Co-authors:}

Héctor Gallardo-Rincón ${ }^{a}$

Alejandra Montoya ${ }^{a}$

Luis Alberto Martínez-Juárez $z^{\mathrm{a}, \mathrm{b}}$

Julieta Lomelín-Gascón ${ }^{a}$

Rodrigo Saucedo-Martínez ${ }^{\mathrm{a}}$

Ricardo Mújica-Rosales ${ }^{a}$

Roberto Tapia-Conyera,c

\section{Affiliations:}

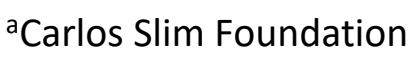

${ }^{b}$ London School of Hygiene and Tropical Medicine

'National Autonomous University of Mexico

All authors have no conflicts of interest 


\section{BACKGROUND / OBJECTIVE}

\section{Background}

The burden of non-communicable diseases (NCDs) has increased globally in recent years, particularly in Mexico, and there is a strong relation between NCDs and the severity and mortality of SARS-CoV-2 infection. However, the separation of health care resources for NCDs versus infectious disease in Mexico has created a situation where a syndemic of chronic NCDs and COVID-19 may thrive if not addressed.

The timely and correct management of chronic diseases such as Diabetes is essential during the COVID-19 pandemic. Several studies have reported an association between the severity of COVID-19 and Diabetes, as well as hypertension, cardiovascular disease and obesity. ${ }^{1-6}$

Though current evidence does not suggest an increased risk of COVID-19 infection for those with Diabetes (type 1 and 2 Diabetes), studies do describe that Diabetes is related to a poor prognosis for the COVID-19 disease. ${ }^{3,5-7}$
The risk of severe COVID or death in those with Diabetes has been described since the beginning of the pandemic in Wuhan. However, prognosis depends on different factors such as age, sex, other comorbidities like hypertension and obesity, and pro-inflammatory and pro-coagulative states. ${ }^{7}$

Aggressive metabolic control in patients with Diabetes and hypertension can decrease the risk of severe acute respiratory syndrome due to the COVID-19 virus. Therefore, it is essential to improve the management of NCDs at all levels of health service delivery to reduce mortality from COVID.

\section{Objective}

This work evaluates the association between Diabetes and main comorbidities (Obesity, hypertension and Chronic Kidney Disease) on COVID-19 outcomes (prevalence, hospitalization, lethality and hospital fatality) in the Mexican population. 


\section{METHODS AND RESULTS}

\section{Methodology}

We used available public data released by the Mexican COVID-19 surveillance system from January 1 to December 31 of $2020 .^{8}$

We assessed hospitalization and fatality risk for people with self-reported Diabetes and comorbidities, using a multiple logistic regression model adjusted for age, sex, smoking status, and marginalization of residence.
$3,401,172$ People tested
$399,953(11.8 \%)$

had Diabetes

Figure 1. Distribution (\%) by category of age of people with selfreported Diabetes tested for COVID-19 between January and December 2020

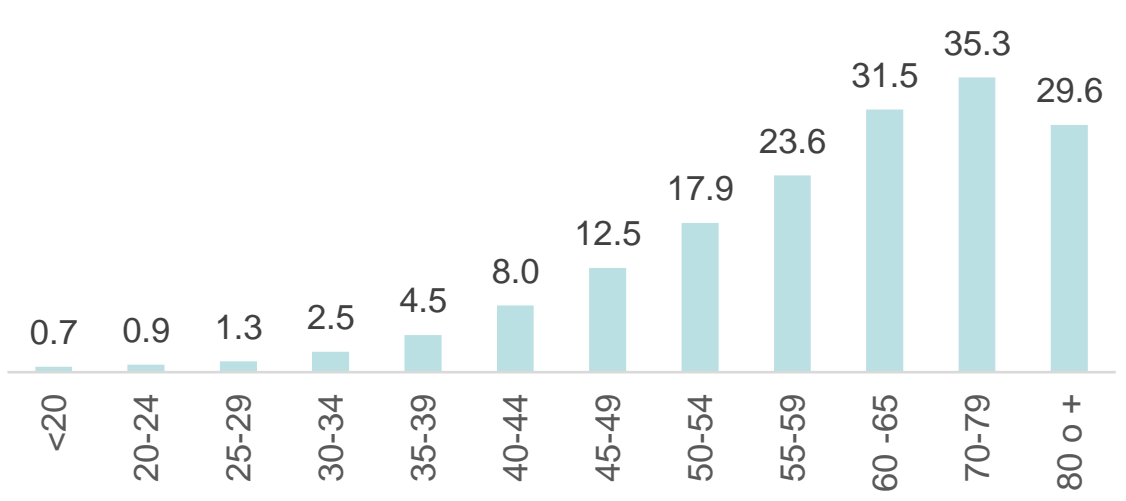




\section{REsULtS}

Figure 2. Comparison of hospitalization and hospital fatality of COVID-19 disease between populations with and without self-reported Diabetes $(N=1,426,094)$.

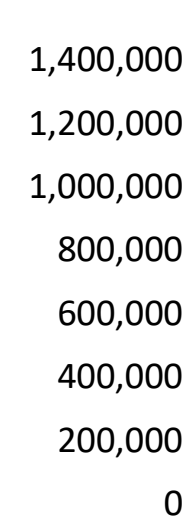

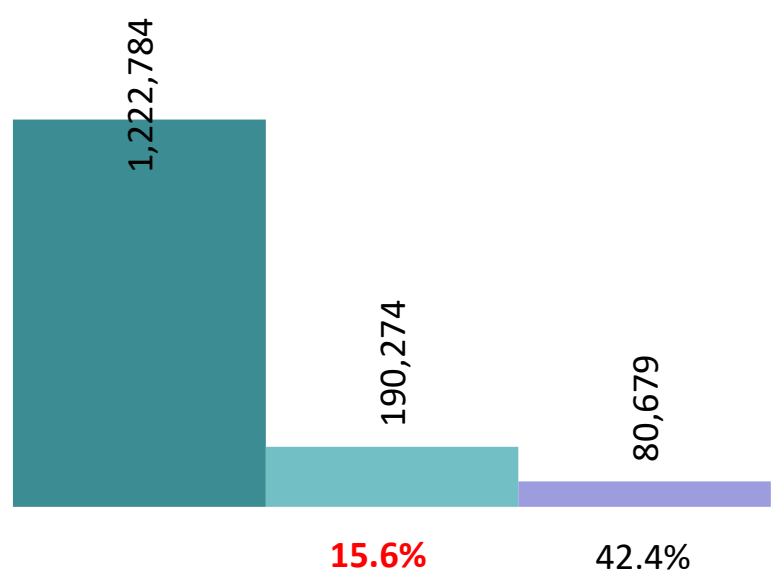

Patients without Diabetes

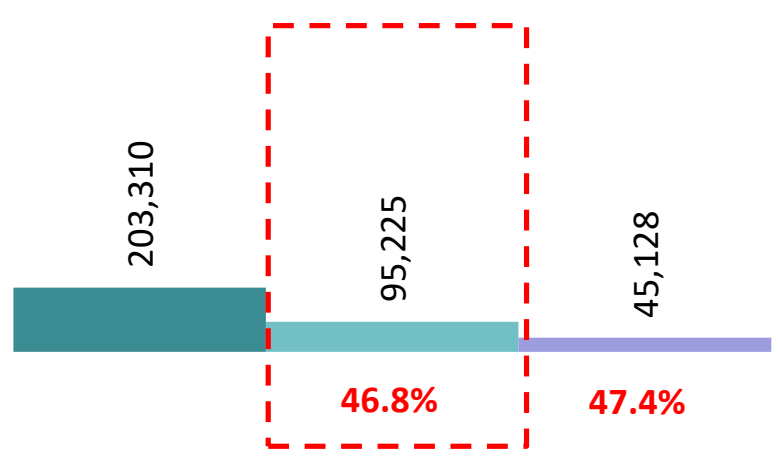

Patients with Diabetes 


\section{RESULTS}

Figure 3. Comparison of main COVID-19 related outcomes among people with self-reported Obesity, Hypertension, Diabetes and Chronic Kidney Disease $(n=1,384,470)$.

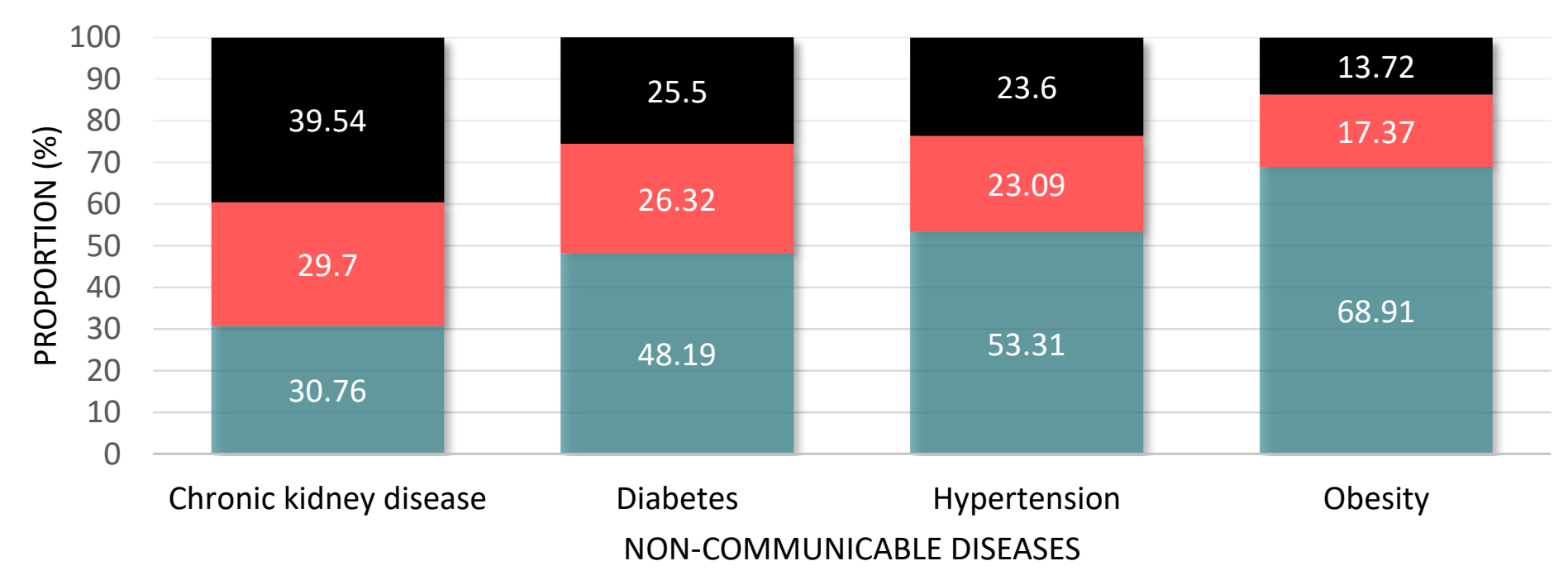




\section{REsULTS}

Figure 4. Distribution of deaths per 100 positive COVID-19 cases among people with self-reported NonCommunicable Diseases $(n=1,384,470)$.

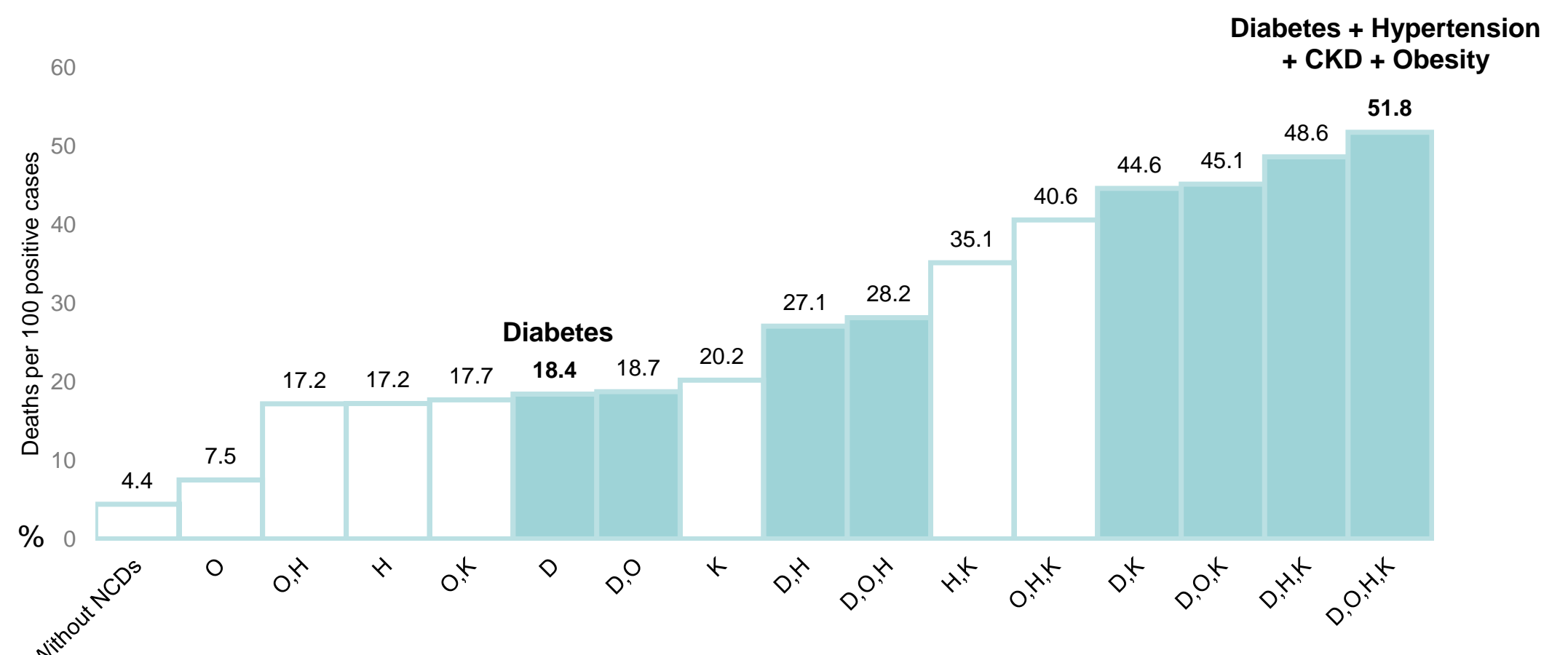


Table 1. Multiple logistic regression model for COVID-19 related outcomes among people with self-reported Diabetes and Non-Communicable Diseases.
Odds Ratios for main COVID-19 related outcomes among people with self-reported Diabetes $(\mathrm{N}=3,401,172)$

\section{Positivity}

Hospitalization

In-hospital letality

Out of hospital letality
OR $(95 \% \mathrm{Cl})$

$1.31(1.28-1.33)$

$2.20(2.10-2.30)$

$1.27(1.27-1.40)$

$1.98(1.90-2.10)$

Odds Ratios for COVID-19 lethality among people with selfOR $\quad(95 \% \mathrm{Cl})$

reported Non-communicable diseases $(\mathrm{N}=1,384,470)$

\section{Hypertension}

Obesity

Hypertension + Obesity

Diabetes

Diabetes + Hypertension

Diabetes + Obesity

Diabetes + Hypertension+ Obesity

Obesity + Chronic Kidney Disease

Chronic Kidney Disease

Hypertension + Obesity+ Chronic Kidney Disease

Diabetes + Chronic Kidney Disease

Diabetes + Obesity + Chronic Kidney Disease

Diabetes + Hypertension+ Obesity + Chronic Kidney Disease

Diabetes + Hypertension+ Chronic Kidney Disease

Hypertension + Chronic Kidney Disease
$1.42(1.40-1.44)$

$1.73(1.70-1.76)$

$2.08(2.03-2.13)$

$2.26(2.22-2.30)$

$2.62(2.57-2.67)$

$3.12(3.02-3.22)$

$3.32(3.23-3.40)$

$3.65(3.09-4.31)$

$4.50(4.21-4.82)$

$6.41(5.51-7.46)$

$7.31(6.55-8.16)$

$8.02(6.45-9.97)$

$8.99(8.20-9.85)$

$9.62(9.11-10.17)$

$9.75(9.08-10.46)$ 


\section{CONCLUSION}

Of the 3,401,172 people analyzed, Diabetes (DM) was presented in 399,953 (11.8\%). In this population, DM and hypertension (HT) was present in 191,178 (47.8\%), DM and Obesity in 35,996 (9.0\%), and DM with Chronic Kidney Diseases (CKD) in 27,997 (7.0\%).

When comparing the frequency of hospitalization and death due to COVID-19 between people with and without DM, it is important to stress that the frequency of hospitalization in non-diabetics was 190,274 (15.5\%), and for death was 80,679 (42.4\%); however, these outcomes had a higher frequency among diabetics, finding a frequency of hospitalization of $95,225(46.8 \%)$, and $45,128(47.4 \%)$ for death.

When analyzing the mortality due to COVID-19 per 100 positive cases according to the different combinations of comorbidities [Diabetes, Hypertension, Obesity and CKD], the proportion of deaths for those with DM, HT and CKD was $51.8 \%$; for DM, Obesity and HT was $18.5 \%$; and for only DM was $18.4 \%$. These results suggest that the presence of additional comorbidities in patients with DM should be considered of a high risk for death from COVID-19.

4 Finally, people with DM had significant $(p<.005)$ higher odds of hospitalization OR:2.2, hospital 1.27 and non-hospital 1.98 fatality. In addition, people with DM and CKD had the highest odds of hospitalization 7.3 died in hospital (2.14) or out of hospital (6.5) compared with cases without DM.

This analysis points out that Diabetes contributes to the risk of infection and worse outcomes for those infected by SARS-CoV-2. More must be done to prevent and control Diabetes and comorbidities to reduce the burden of COVID-19 outcomes. 


\section{RefERENCES}

1 Huang I, Lim MA, Pranata R. Diabetes mellitus is associated with increased mortality and severity of disease in COVID-19 pneumonia - A systematic review, meta-analysis, and meta-regression: Diabetes and COVID-19. Diabetes Metab Syndr Clin Res Rev 2020; 14: 395-403.

2 Zhu L, She ZG, Cheng X, et al. Association of Blood Glucose Control and Outcomes in Patients with COVID-19 and Pre-existing Type 2 Diabetes. Cell Metab 2020; 31: 1068-1077.e3.

3 Petrilli CM, Jones SA, Yang J, et al. Factors associated with hospital admission and critical illness among 5279 people with coronavirus disease 2019 in New York City: Prospective cohort study. BMJ 2020; 369. DOI:10.1136/bmj.m1966.

4 Zhou F, Yu T, Du R, et al. Clinical course and risk factors for mortality of adult inpatients with COVID-19 in Wuhan, China: a retrospective cohort study. Lancet 2020; 395: 1054-62.

5 Li B, Yang J, Zhao F, et al. Prevalence and impact of cardiovascular metabolic diseases on COVID-19 in China. Clin. Res. Cardiol. 2020; 109: 531-8.

6 Guo L, Shi Z, Zhang Y, et al. Comorbid Diabetes and the risk of disease severity or death among 8807 COVID-19 patients in China: A meta-analysis. Diabetes Res Clin Pract 2020; 166: 108346.

7 Apicella M, Campopiano MC, Mantuano M, Mazoni L, Coppelli A, Del Prato S. COVID-19 in people with Diabetes: understanding the reasons for worse outcomes. Lancet Diabetes Endocrinol. 2020; 8: 782-92.

8 Gobierno de México. Coronavirus - gob.mx. https://coronavirus.gob.mx/\# (accessed May 25, 2021). 